\title{
Manipulations of Submicro-fibers of Culex Pipiens with the Help of Nano-tweezers with Shape Memory Effect into Vacuum Chamber of FIB.
}

\author{
Alexander Kamantsev ${ }^{1}$, Alexey Mashirov ${ }^{1}$, Pavel Mazaev ${ }^{1}$, Victor Koledov ${ }^{1}$, Vladimir Shavrov ${ }^{1}$, \\ Vladimir Dikan ${ }^{2}$, Artemy Irzhak ${ }^{2}$, Alexander Shelyakov ${ }^{3}$ \\ 1. Kotelnikov Institute of Radio-engineering and Electronics of RAS, Moscow, Russia. \\ 2. National University of Science and Technology "MISIS", Moscow, Russia. \\ 3. National Research Nuclear University "MEPhI", Moscow, Russia.
}

In recent years, a record small mechanical tools based on composites with shape memory effect (SME) were created [1-4]. Application of the technology of selective ion etching allowed for the creation of two-layer composite actuators and tools based on rapidly quenched nonmagnetic alloys with SME, such as $\mathrm{Ti}_{2} \mathrm{NiCu}$ [1]. These composite actuators can change their shape reversely and produce mechanical work using only "one-way" SME of the alloy [2]. Currently in the field of manipulation and manufacturing at the nanoscale, there is an urgent need to develop new functional materials in order to fill the gap between the dimensions of modern MEMS and real size of nano-objects to be manipulated. Recently the operation of nano-tweezers using layered composites based on alloy with SME driven by thermal actuation has been demonstrated [1-3]. The operation of the $\mathrm{Ti}_{2} \mathrm{NiCu} / \mathrm{Pt}$ composite actuator driven by heating was demonstrated, with an overall volume of the actuator of less than $1 \mu \mathrm{m}^{3}$ and thickness of active layer of the $\mathrm{T}_{2} \mathrm{NiCu}$ alloy being down to $70 \mathrm{~nm}$ [1].

These achievements of nanotechnology can be used certainly in various branches of biology. Developed technique allow to hold, move and manipulate of animate or inanimate objects of submicron and nanometer sizes, for example, carbon nanotubes, graphene sheets, bacteria, viruses, biological particles of different nature. The purpose of this paper is description of experiments on preparation of insect's fibers of submicron sizes into a vacuum chamber of FIB.

The object of the study served as Culex pipiens. This species is distributed universally and has a large epidemic importance. The experiment consisted in the selection of a part of fiber, its separation from the insect's body (Fig. 1), displacement and attaching it on a copper grid (Fig. 2) for further TEM studies. The experiment was done into vacuum chamber of FEI Strata 201 FIB device with the help of composite nano-tweezers with SME. For positioning in space the nano-tweezers was attached to needle of Omniprobe micromanipulator (by CVD process). The whole process of manipulations and the characteristic scales are presented on Fig. 1 and Fig. 2. We are demonstrating on example of manipulations of insect's fiber that such microinstruments might help to resolve some unresolved problems of medicine and biotechnology, particularly in single cell manipulation, microsurgery, drug delivery systems, etc.

[1] D. Zakharov, G. Lebedev, A. Irzhak, et al., Smart Matr. Struct. 21, (2012), p. 052001.

[2] A. Irzhak, V. Kalashnikov, V. Koledov, et al., Techn. Phys. Lett. 36, (2010), p. 329.

[3] A.V. Shelyakov, N.N. Sitnikov, A.P. Menushenkov et al., Thin. Sol. Films 519, (2011), p. 5314.

[4] E. Kalimullina, A. Kamantsev, V. Koledov, et al., Phys. Stat. Solidi (C) 11, 5-6, (2014), p. 1023.

[5] The authors acknowledge funding from the Russian Sciences Foundation, Grant № 14-19-01644. 


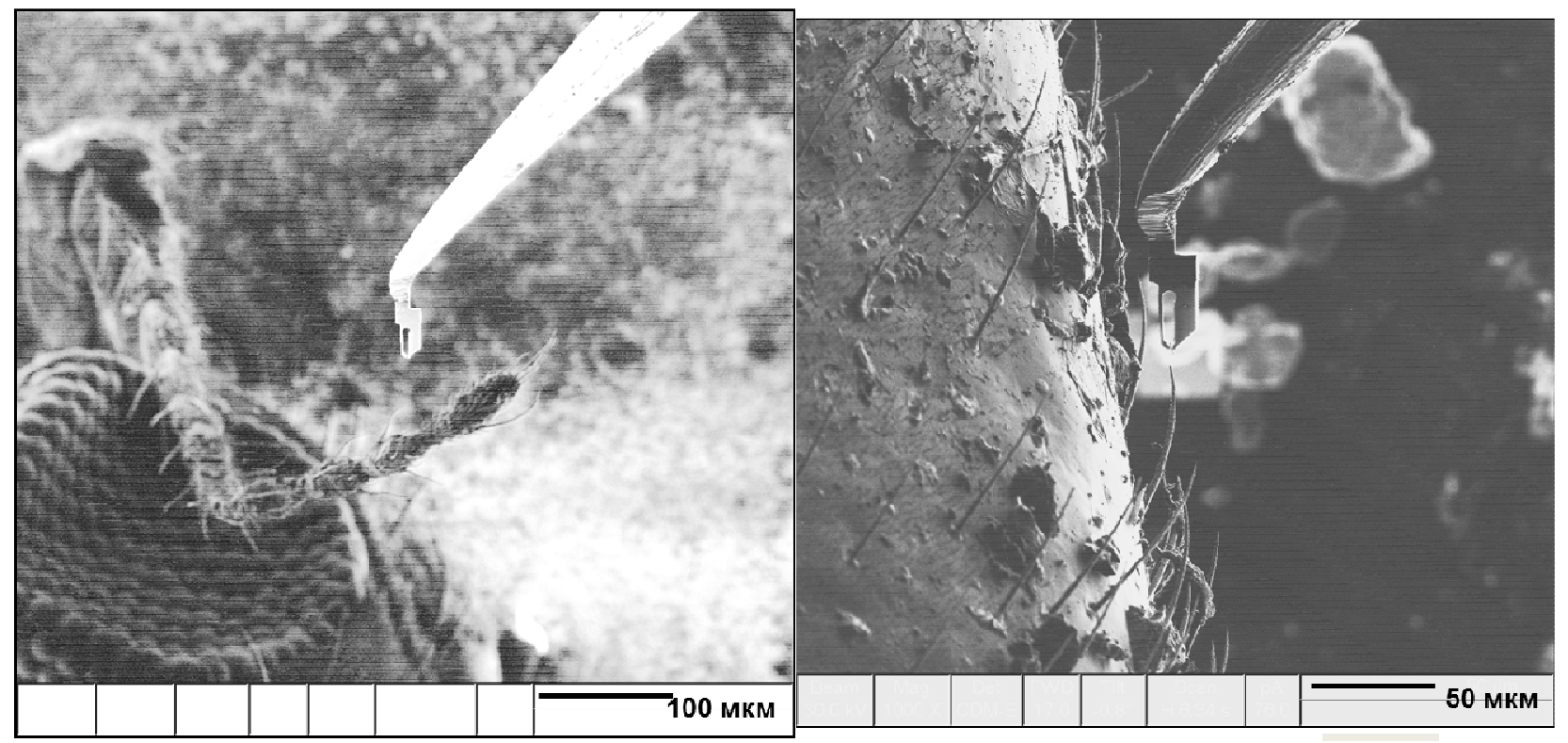

Figure 1. Feeding of nano-tweezers and selection of a part of fiber on body of Culex pipiens.

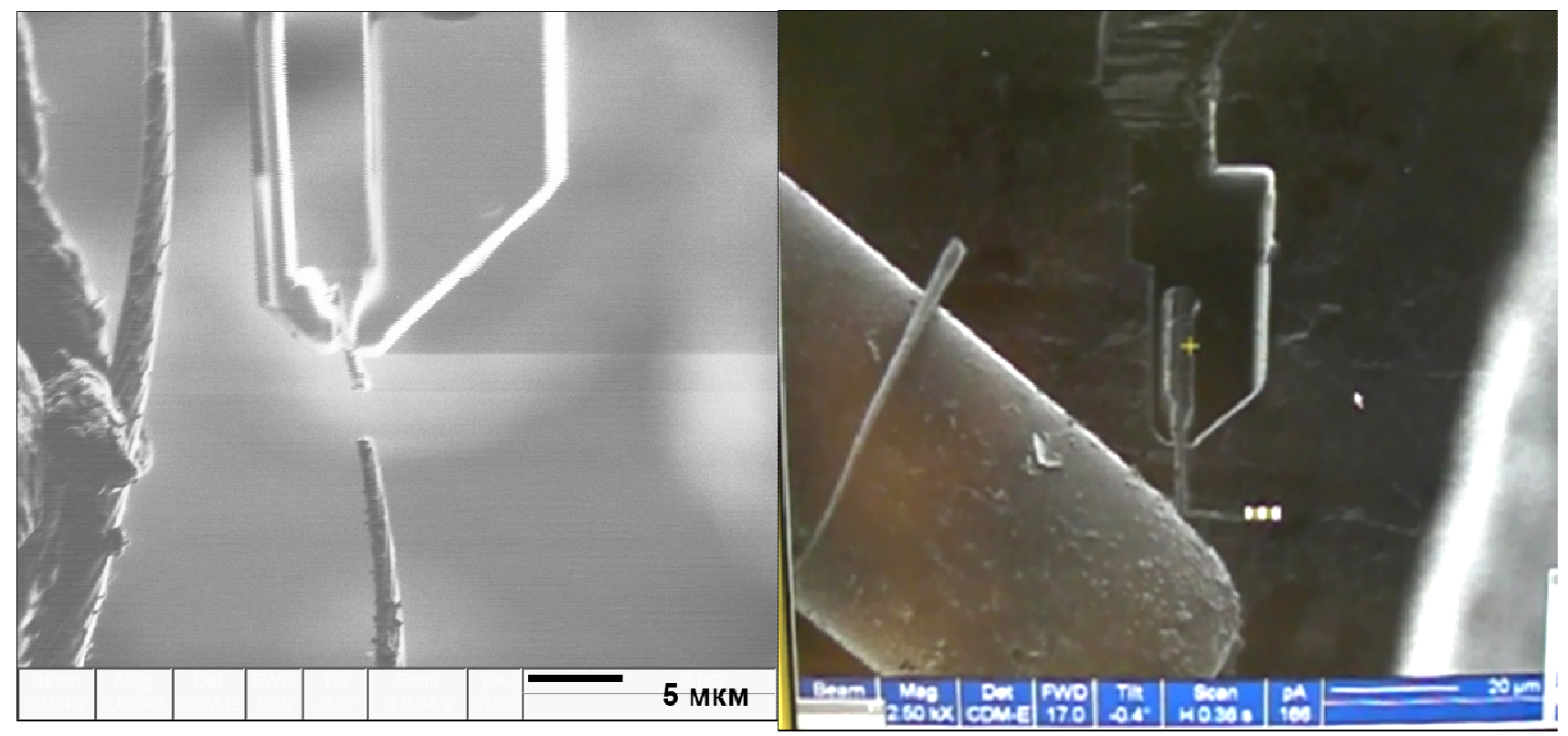

Figure 2. Separation of part of fiber from Culex pipiens body, displacement and attaching it on copper grid. 